\title{
Note on irreducible approach to reducible second-class constraints
}

\author{
C. Bizdadea, A. Constantin, S. O. Saliu ${ }^{\dagger}$ \\ Department of Physics, University of Craiova \\ 13 A. I. Cuza Str., Craiova RO-1100, Romania
}

November 21, 2018

\begin{abstract}
An irreducible canonical approach to reducible second-class constraints is given. The procedure is illustrated on gauge-fixed twoforms.
\end{abstract}

PACS number: 11.10.Ef

The canonical approach to systems with reducible second-class constraints is quite intricate, demanding a modification of the usual rules as the matrix of the Poisson brackets among the constraints is not invertible. Thus, it is necessary to isolate a set of independent constraints, and then construct the Dirac bracket [1, 目] with respect to this set. The split of the constraints may lead to the loss of important symmetries, so it should be avoided. As shown in [3, 4, 5, 6, 7], it is however possible to construct the Dirac bracket in terms of a noninvertible matrix without separating the independent constraint functions. A third possibility is to substitute the reducible secondclass constraints by some irreducible ones and further work with the Dirac bracket based on the irreducible constraints. This idea has been suggested in [8] mainly in the context of two- and three-form gauge fields.

*e-mail address: bizdadea@central.ucv.ro

†e-mail address: osaliu@central.ucv.ro 
Although the idea based on irreducible second-class constraints is known, a general irreducible procedure equivalent to the reducible one has not been developed so far. This is the aim of this letter.

We start with a system locally described by $N$ canonical pairs $z^{a}=$ $\left(q^{i}, p_{i}\right)$, subject to the second-class constraints

$$
\chi_{\alpha_{0}}\left(z^{a}\right) \approx 0, \alpha_{0}=1, \cdots, M_{0} .
$$

For simplicity, we take all the phase-space variables to be bosonic. In addition, we presume that the functions $\chi_{\alpha_{0}}$ are not independent, there existing some nonvanishing functions $Z_{\alpha_{1}}^{\alpha_{0}}$ such that

$$
Z_{\alpha_{1}}^{\alpha_{0}} \chi_{\alpha_{0}}=0, \alpha_{1}=1, \cdots, M_{1} \text {. }
$$

Moreover, we assume that $Z_{\alpha_{1}}^{\alpha_{0}}$ are independent and (2) are the only reducibility relations with respect to the constraints (11). These constraints are purely second-class if any maximal, independent set of $M_{0}-M_{1}$ constraint functions $\chi_{A}\left(A=1, \cdots, M_{0}-M_{1}\right)$ among the $\chi_{\alpha_{0}}$ is such that the matrix

$$
C_{A B}=\left[\chi_{A}, \chi_{B}\right]
$$

is invertible. In terms of independent constraints, the Dirac bracket takes the form

$$
[F, G]^{*}=[F, G]-\left[F, \chi_{A}\right] M^{A B}\left[\chi_{B}, G\right],
$$

where $M^{A B} C_{B C} \approx \delta_{C}^{A}$. We can rewrite the Dirac bracket in (州) without finding a definite subset of independent second-class constraints as follows. We start with the matrix

$$
C_{\alpha_{0} \beta_{0}}=\left[\chi_{\alpha_{0}}, \chi_{\beta_{0}}\right]
$$

that is not invertible because

$$
Z_{\alpha_{1}}^{\alpha_{0}} C_{\alpha_{0} \beta_{0}} \approx 0
$$

If $d_{\alpha_{0}}^{\alpha_{1}}$ is solution to the equation

$$
d_{\alpha_{0}}^{\alpha_{1}} Z_{\beta_{1}}^{\alpha_{0}} \approx \delta_{\beta_{1}}^{\alpha_{1}}
$$

then we can introduce a matrix [6] $M^{\alpha_{0} \beta_{0}}$ through the relation

$$
M^{\alpha_{0} \beta_{0}} C_{\beta_{0} \gamma_{0}} \approx \delta_{\gamma_{0}}^{\alpha_{0}}-Z_{\alpha_{1}}^{\alpha_{0}} d_{\gamma_{0}}^{\alpha_{1}}
$$


with $M^{\alpha_{0} \beta_{0}}=-M^{\beta_{0} \alpha_{0}}$. Then, the formula [6]

$$
[F, G]^{*}=[F, G]-\left[F, \chi_{\alpha_{0}}\right] M^{\alpha_{0} \beta_{0}}\left[\chi_{\beta_{0}}, G\right],
$$

defines the same Dirac bracket like (4) on the surface (1).

After this brief review on the Dirac bracket for reducible second-class constraints, we pass to the implementation of our irreducible procedure. The solution to the equation (面) has the form

$$
d_{\alpha_{0}}^{\alpha_{1}}=\left(\delta_{\beta_{1}}^{\alpha_{1}}+m_{\beta_{1}}^{\alpha_{1} \beta_{0}} \chi_{\beta_{0}}\right) \bar{D}_{\gamma_{1}}^{\beta_{1}} A_{\alpha_{0}}^{\gamma_{1}},
$$

where $A_{\alpha_{0}}^{\gamma_{1}}$ are some functions chosen such that

$$
\operatorname{rank}\left(D_{\alpha_{1}}^{\gamma_{1}}\right) \equiv \operatorname{rank}\left(Z_{\alpha_{1}}^{\alpha_{0}} A_{\alpha_{0}}^{\gamma_{1}}\right)=M_{1}
$$

$\bar{D}_{\gamma_{1}}^{\beta_{1}}$ stands for the inverse of $D_{\alpha_{1}}^{\gamma_{1}}$, and $m_{\beta_{1}}^{\alpha_{1} \beta_{0}}$ are some arbitrary functions. Inserting (10) in (8) we find

$$
M^{\alpha_{0} \beta_{0}} C_{\beta_{0} \gamma_{0}} \approx D_{\gamma_{0}}^{\alpha_{0}}
$$

with

$$
D_{\gamma_{0}}^{\alpha_{0}}=\delta_{\gamma_{0}}^{\alpha_{0}}-Z_{\alpha_{1}}^{\alpha_{0}} \bar{D}_{\gamma_{1}}^{\alpha_{1}} A_{\gamma_{0}}^{\gamma_{1}}
$$

With these elements at hand, the next theorem is shown to hold.

Theorem 1 There exists an invertible antisymmetric matrix $\mu^{\alpha_{0} \beta_{0}}$ such that the Dirac bracket (9) takes the form

$$
[F, G]^{*}=[F, G]-\left[F, \chi_{\alpha_{0}}\right] \mu^{\alpha_{0} \beta_{0}}\left[\chi_{\beta_{0}}, G\right],
$$

on the surface (1).

Proof. First, we observe that the matrix (13) is a projector

$$
D_{\gamma_{0}}^{\alpha_{0}} D_{\lambda_{0}}^{\gamma_{0}}=D_{\lambda_{0}}^{\alpha_{0}}
$$

and satisfies the relations

$$
\begin{aligned}
& D_{\gamma_{0}}^{\alpha_{0}} Z_{\gamma_{1}}^{\gamma_{0}}=0, \\
& A_{\alpha_{0}}^{\gamma_{1}} D_{\gamma_{0}}^{\alpha_{0}}=0,
\end{aligned}
$$




$$
D_{\gamma_{0}}^{\alpha_{0}} \chi_{\alpha_{0}}=\chi_{\gamma_{0}}
$$

Multiplying (12) by $A_{\alpha_{0}}^{\gamma_{1}}$ and using (17) we obtain the relations $A_{\alpha_{0}}^{\gamma_{1}} M^{\alpha_{0} \beta_{0}} C_{\beta_{0} \gamma_{0}} \approx$ 0 , which then lead to

$$
A_{\alpha_{0}}^{\gamma_{1}} M^{\alpha_{0} \beta_{0}} \approx 0
$$

The relations (19) allow us to represent $M^{\alpha_{0} \beta_{0}}$ under the form

$$
M^{\alpha_{0} \beta_{0}} \approx D_{\lambda_{0}}^{\alpha_{0}} \mu^{\lambda_{0} \sigma_{0}} D_{\sigma_{0}}^{\beta_{0}}
$$

where $\mu^{\lambda_{0} \sigma_{0}}$ is an antisymmetric matrix. Now, we prove that $\mu^{\lambda_{0} \sigma_{0}}$ is invertible. On account of (16), the solution to (20) reads as

$$
\mu^{\lambda_{0} \sigma_{0}} \approx M^{\lambda_{0} \sigma_{0}}+Z_{\lambda_{1}}^{\lambda_{0}} \bar{D}_{\beta_{1}}^{\lambda_{1}} \omega^{\beta_{1} \gamma_{1}} \bar{D}_{\gamma_{1}}^{\sigma_{1}} Z_{\sigma_{1}}^{\sigma_{0}}
$$

for an invertible antisymmetric matrix $\omega^{\beta_{1} \gamma_{1}}$. As the only null vectors of $M^{\alpha_{0} \beta_{0}}$ are $A_{\alpha_{0}}^{\gamma_{1}}($ see $(19))$, it results that $A_{\lambda_{0}}^{\rho_{1}} \mu^{\lambda_{0} \sigma_{0}} \approx \omega^{\rho_{1} \gamma_{1}} \bar{D}_{\gamma_{1}}^{\sigma_{1}} Z_{\sigma_{1}}^{\sigma_{0}}$ vanish if and only if $Z_{\sigma_{1}}^{\sigma_{0}}$ vanish (because $\omega^{\rho_{1} \gamma_{1}} \bar{D}_{\gamma_{1}}^{\sigma_{1}}$ is invertible). However, by assumption we have that not all $Z_{\sigma_{1}}^{\sigma_{0}}$ vanish, so it results that $\omega^{\rho_{1} \gamma_{1}} \bar{D}_{\gamma_{1}}^{\sigma_{1}} Z_{\sigma_{1}}^{\sigma_{0}}$ is nonvanishing. In consequence, $\mu^{\lambda_{0} \sigma_{0}}$ has no null vectors, being therefore invertible. Inserting (20) in (9) and using (18), we deduce precisely (14). This proves the theorem.

Formulas (12) and (20) allow us to represent $C_{\beta_{0} \gamma_{0}}$ like

$$
C_{\beta_{0} \gamma_{0}} \approx D_{\beta_{0}}^{\rho_{0}} \mu_{\rho_{0} \tau_{0}} D_{\gamma_{0}}^{\tau_{0}}
$$

which gives

$$
\mu_{\rho_{0} \tau_{0}} \approx C_{\rho_{0} \tau_{0}}+A_{\rho_{0}}^{\rho_{1}} \omega_{\rho_{1} \tau_{1}} A_{\tau_{0}}^{\tau_{1}},
$$

where $\mu_{\rho_{0} \tau_{0}}$ and $\omega_{\rho_{1} \tau_{1}}$ stand for the inverses of the corresponding upper-indices matrices. It is easy to see that (20) and (22) verify (12). Apart from being antisymmetric and invertible, the matrix $\omega_{\rho_{1} \tau_{1}}$ is up to our choice. In order to endow this matrix with a concrete significance, we introduce some new variables $\left(y_{\alpha_{1}}\right)_{\alpha_{1}=1, \cdots, M_{1}}$ with the Poisson brackets

$$
\left[y_{\alpha_{1}}, y_{\beta_{1}}\right]=\omega_{\alpha_{1} \beta_{1}}
$$

and consider the system subject to the reducible second-class constraints

$$
\chi_{\alpha_{0}} \approx 0, y_{\alpha_{1}} \approx 0
$$


The Dirac bracket on the phase-space described by $\left(z^{a}, y_{\alpha_{1}}\right)$ corresponding to the above second-class constraints reads as

$$
\left.[F, G]^{*}\right|_{z, y}=[F, G]-\left[F, \chi_{\alpha_{0}}\right] \mu^{\alpha_{0} \beta_{0}}\left[\chi_{\beta_{0}}, G\right]-\left[F, y_{\alpha_{1}}\right] \omega^{\alpha_{1} \beta_{1}}\left[y_{\beta_{1}}, G\right]
$$

where the Poisson brackets from the right hand-side of (26) contain derivatives with respect to all $z^{a}$ and $y_{\alpha_{1}}$. After some computation we infer that

$$
\left.[F, G]^{*}\right|_{z, y} \approx[F, G]^{*},
$$

where $[F, G]^{*}$ is given by (14) and the weak equality refers to the surface (25). Under these considerations, we are able to prove the following theorem.

Theorem 2 (i) There exist some irreducible second-class constraints equivalent to (25)

$$
\tilde{\chi}_{\alpha_{0}}\left(z^{a}, y_{\alpha_{1}}\right) \approx 0
$$

such that

$$
\left[\tilde{\chi}_{\alpha_{0}}, \tilde{\chi}_{\beta_{0}}\right] \approx \mu_{\alpha_{0} \beta_{0}}
$$

(ii) The Dirac bracket with respect to the irreducible second-class constraints (28)

$$
\left.[F, G]^{*}\right|_{\text {ired }}=[F, G]-\left[F, \tilde{\chi}_{\alpha_{0}}\right] \mu^{\alpha_{0} \beta_{0}}\left[\tilde{\chi}_{\beta_{0}}, G\right],
$$

coincides with (20)

$$
\left.[F, G]^{*}\right|_{\text {ired }}=\left.[F, G]^{*}\right|_{z, y},
$$

on the surface (28).

Proof. (i) Using (23), formulas (29) become

$$
\left[\tilde{\chi}_{\alpha_{0}}, \tilde{\chi}_{\beta_{0}}\right] \approx C_{\alpha_{0} \beta_{0}}+A_{\alpha_{0}}^{\rho_{1}} \omega_{\rho_{1} \tau_{1}} A_{\beta_{0}}^{\tau_{1}} \text {. }
$$

Now, we prove that the solution to the above equations is expressed by

$$
\tilde{\chi}_{\alpha_{0}}=\chi_{\alpha_{0}}+A_{\alpha_{0}}^{\alpha_{1}} y_{\alpha_{1}}
$$

The functions $\tilde{\chi}_{\alpha_{0}}$ are irreducible. Indeed, $Z_{\beta_{1}}^{\alpha_{0}} \tilde{\chi}_{\alpha_{0}}=D_{\beta_{1}}^{\alpha_{1}} y_{\alpha_{1}}$ vanish if and only if $y_{\alpha_{1}}$ vanish, so if and only if the new constraints reduce to (25). This 
proves the irreducibility. After some simple computation, from (33) we infer that

$$
\chi_{\alpha_{0}}=D_{\alpha_{0}}^{\beta_{0}} \tilde{\chi}_{\beta_{0}}, y_{\alpha_{1}}=\bar{D}_{\alpha_{1}}^{\beta_{1}} Z_{\beta_{1}}^{\beta_{0}} \tilde{\chi}_{\beta_{0}} .
$$

It is easy to see that if (25) hold, then (28) also hold (with $\tilde{\chi}_{\alpha_{0}}$ given by (33)). From (34) we obtain that if (28) hold, (25) hold, too, so

$$
\tilde{\chi}_{\alpha_{0}} \approx 0 \Leftrightarrow \chi_{\alpha_{0}} \approx 0, y_{\alpha_{1}} \approx 0
$$

such that the constraints (28) are equivalent to (25). Finally, if we use (35), then the functions (33) satisfy (32). This proves (i).

(ii) By direct computation we get

$$
\begin{aligned}
& {\left.[F, G]^{*}\right|_{\text {ired }} \approx[F, G]^{*}-\left[F, \chi_{\alpha_{0}}\right] \mu^{\alpha_{0} \beta_{0}} A_{\beta_{0}}^{\beta_{1}}\left[y_{\beta_{1}}, G\right]-} \\
& {\left[F, y_{\alpha_{1}}\right] A_{\alpha_{0}}^{\alpha_{1}} \mu^{\alpha_{0} \beta_{0}}\left[\chi_{\beta_{0}}, G\right]-\left[F, y_{\alpha_{1}}\right] A_{\alpha_{0}}^{\alpha_{1}} \mu^{\alpha_{0} \beta_{0}} A_{\beta_{0}}^{\beta_{1}}\left[y_{\beta_{1}}, G\right] .}
\end{aligned}
$$

On the other hand, from (21) we obtain that

$$
\begin{gathered}
\mu^{\alpha_{0} \beta_{0}} A_{\beta_{0}}^{\beta_{1}} \approx Z_{\alpha_{1}}^{\alpha_{0}} \bar{D}_{\gamma_{1}}^{\alpha_{1}} \omega^{\gamma_{1} \beta_{1}}, A_{\alpha_{0}}^{\alpha_{1}} \mu^{\alpha_{0} \beta_{0}} \approx \omega^{\alpha_{1} \gamma_{1}} \bar{D}_{\gamma_{1}}^{\beta_{1}} Z_{\beta_{1}}^{\beta_{0}}, \\
A_{\alpha_{0}}^{\alpha_{1}} \mu^{\alpha_{0} \beta_{0}} A_{\beta_{0}}^{\beta_{1}} \approx \omega^{\alpha_{1} \beta_{1}} .
\end{gathered}
$$

Inserting (37 38) in (36), we immediately find (31). This proves (ii).

The last theorem proves that we can approach reducible second-class constraints in an irreducible fashion. Thus, starting with the reducible constraints (11) we construct the irreducible constraint functions (33), whose Poisson brackets form an invertible matrix. Formulas (27) and (31) ensure that $\left.[F, G]^{*}\right|_{\text {ired }} \approx[F, G]^{*}$, so the fundamental Dirac brackets among the original variables $z^{a}$ within the irreducible setting coincide with those from the reducible version

$$
\left.\left[z^{a}, z^{b}\right]^{*}\right|_{\text {ired }} \approx\left[z^{a}, z^{b}\right]^{*}
$$

Moreover, the new variables $y_{\alpha_{1}}$ do not affect the irreducible Dirac bracket as from $(31)$ we have that $\left.\left[y_{\alpha_{1}}, F\right]^{*}\right|_{\text {ired }} \approx 0$. Thus, the equations of motion for the original reducible system can be written as $\left.\dot{z}^{a} \approx\left[z^{a}, H\right]^{*}\right|_{\text {ired }}$, where $H$ is the canonical Hamiltonian. The equations of motion for $y_{\alpha_{1}}$ read as $\dot{y}_{\alpha_{1}} \approx 0$, and lead to $y_{\alpha_{1}}=0$ by taking some appropriate boundary conditions (vacuum to vacuum) for these unphysical variables. This completes the general procedure. 
Let us briefly exemplify the general theory on gauge-fixed two-forms, subject to the second-class constraints

$$
\chi_{\alpha_{0}} \equiv\left(\begin{array}{c}
-2 \partial^{k} \pi_{k i} \\
-\partial_{l} A^{l j}
\end{array}\right) \approx 0 .
$$

The constraints involving the temporal components of the two-form and its momenta are irreducible, and will be omitted. The constraints (40) are firststage reducible, with the reducibility functions expressed by

$$
Z_{\alpha_{1}}^{\alpha_{0}}=\left(\begin{array}{cc}
\partial^{i} & 0 \\
0 & \partial_{j}
\end{array}\right)
$$

Acting along the line exposed in the above, we take the matrix $A_{\alpha_{0}}^{\alpha_{1}}$ under the form

$$
A_{\alpha_{0}}^{\alpha_{1}}=\left(\begin{array}{cc}
-\partial_{i} & 0 \\
0 & -\partial^{j}
\end{array}\right)
$$

SO

$$
D_{\beta_{1}}^{\alpha_{1}}=\left(\begin{array}{cc}
-\partial_{i} \partial^{i} & 0 \\
0 & -\partial^{j} \partial_{j}
\end{array}\right),
$$

is invertible. In order to construct the irreducible second-class constraints, we introduce the variables

$$
y_{\alpha_{1}}=\left(\begin{array}{c}
\pi \\
\varphi
\end{array}\right)
$$

and take

$$
\omega_{\alpha_{1} \beta_{1}}=\left(\begin{array}{cc}
0 & -1 \\
1 & 0
\end{array}\right) .
$$

As can be seen, the supplementary scalar fields $(\pi, \varphi)$ are canonically conjugated, with $\pi$ the momentum. Then, the irreducible second-class constraints are expressed by

$$
\tilde{\chi}_{\alpha_{0}} \equiv\left(\begin{array}{c}
-2 \partial^{k} \pi_{k i}-\partial_{i} \pi \\
-\partial_{l} A^{l j}-\partial^{j} \varphi
\end{array}\right) \approx 0,
$$

such that

$$
\mu_{\alpha_{0} \beta_{0}}=\left(\begin{array}{cc}
0 & \delta_{i}{ }^{j} \triangle \\
-\delta^{k}{ }_{l} \triangle & 0
\end{array}\right)
$$


where $\triangle=\partial^{l} \partial_{l}$. By inverting (47) we obtain that the only nonvanishing irreducible Dirac brackets are given by

$$
\left.\left[A^{i j}(x), \pi_{k l}(y)\right]^{*}\right|_{\text {ired }}=\frac{1}{2}\left(\delta^{[i}{ }_{k} \delta_{l}^{j]}+\frac{1}{\triangle} \partial^{[i}{ }^{j]}{ }_{p} \partial_{[k} \delta^{p}{ }_{l]}\right) \delta^{3}(\mathbf{x}-\mathbf{y}),
$$

where the notation $\left[i_{1} \cdots i_{n}\right]$ means antisymmetry with respect to the indices between brackets. The result given by (48) reproduces the standard result from the literature [8, 9]. By means of (48) and of the canonical Hamiltonian associated with the model under study we can immediately write down the corresponding equations of motion. This completes the analysis of the example.

To conclude with, in this paper we have exposed an irreducible procedure for approaching systems with reducible second-class constraints. Our strategy includes three main steps. First, we express the Dirac bracket for the reducible system in terms of an invertible matrix. Second, we establish the equality between this Dirac bracket and that corresponding to the intermediate theory, based on the constraints (25). Third, we prove that there exists an irreducible second-class constraint set equivalent with (25) such that the corresponding Dirac brackets coincide. These three steps enforce the fact that the fundamental Dirac brackets with respect to the original variables derived within the irreducible and original reducible settings coincide. Moreover, the newly added variables do not affect the Dirac bracket, so the canonical approach to the initial reducible system can be developed in terms of the Dirac bracket corresponding to the irreducible theory. Finally, the general procedure was exemplified for gauge-fixed two-forms.

\section{Acknowledgment}

Two of the authors (C.B. and S.O.S.) acknowledge financial support from a Romanian National Council for Academic Scientific Research (CNCSIS) grant.

\section{References}

[1] P. A. M. Dirac, Can. J. Math. 2 (1950) 129 
[2] P. A. M. Dirac, Lectures on Quantum Mechanics (Academic Press, New York, 1967)

[3] L. Brink, J. H. Schwarz, Phys. Lett. B100 (1981) 310

[4] I. Bengtsson, M. Cederwall, Gőteborg Preprint 84-21 (1984)

[5] L. Brink, M. Henneaux, C. Teitelboim, Nucl. Phys. B293 (1987) 505

[6] A. Dresse, J. Fisch, M. Henneaux, C. Schomblond, Phys. Lett. B210 (1988) 141

[7] M. Henneaux, C. Teitelboim, Quantization of Gauge Systems (Princeton University Pres, Princeton, 1992)

[8] R. Banerjee, J. Barcelos-Neto, Ann. Phys. (N. Y.) 265 (1998) 134

[9] R. K. Kaul, Phys. Rev. D18 (1978) 1127 